\title{
Comparing Growth and Carcass Traits of Slow Growing Chicken Parents with Pure Egg Type Parents and Commercial Broilers
}

\author{
Musa Sarica ${ }^{1}$, Umut Sami Yamak ${ }^{1 *}$, Mehmet Akif Boz ${ }^{2}$ \\ ${ }^{1}$ Ondokuz Mayis University, Agricultural Faculty, Department of Animal Science Samsun, Turkey \\ ${ }^{2}$ Bozok University, Faculty of Agricultural and Natural Sciences, Department of Animal Science Yozgat, Turkey \\ *e-mail: usyamak@ omu.edu.tr; Tel: +90 (362) 3121919 / 1389; Fax: +90 (362) 4576034
}

\begin{abstract}
In this study, growth and carcass traits of slow growing parents were compared with commercial broilers and pure parents. Two egg type parents and a commercial meat parent were used in crossings. Male-female mixed 144 chickens per genotype were reared on litter in a house divided into $1.5 \times 1.5 \mathrm{~m}$ pens. Live weight, carcass weight, carcass part ratios, abdominal fat and edible inner organ weights were determined in four dam and three sire line chickens. Colour as measured by $\mathrm{L}^{*}, \mathrm{a}^{*}, \mathrm{~b}^{*}$ values and $\mathrm{pH}$ were assessed in meat of thigh and breast. According to growth, feed efficiency, and carcass characteristics, crossbred chickens exhibited values between pure egg parents and broilers.
\end{abstract}

Key words: Slow growing chickens, cross-breeding, meat colour, meat $\mathrm{pH}$, carcass parts

\section{Yavaş Gelişen Etlik Piliç Ebeveynlerinin Büyüme ve Karkas Özelliklerinin Ticari Etlik Piliç ve Saf Yumurtacı Hatlarla Karşılaştırılması}

\section{Özet}

$\mathrm{Bu}$ çalışmada yavaş gelişen ebeveynlerin ticari broiler ve saf hatlar ile büyüme ve karkas özellikleri karşıllaştırılmıştır. Melezlemelerde iki yumurtacı bir etlik ebeveyn kullanılmıştır. Her genotipten erkek-dişi karışık 144 civciv $1.5 \mathrm{x} 1.5 \mathrm{~m}$ ölçülerindeki bölmelerde altlıklı sistemde yetiştirilmiştir. Canlı ağırlık, karkas ağırlığı, karkas parka oranları, abdominal yağ ve yenilebilir iç organ ağırlıkları 4 ana 3 baba hattında belirlenmiştir. But ve göğüs etlerinde et renginin $L^{*}, a^{*}$ ve $b^{*}$ değerleri ile pH belirlenmiştir. Büyüme, yemden yararlanma oranı ve karkas özelliklerine göre, melez genotipler saf yumurtacı hatlar ile etlik piliçler arasında değerlere sahip olmuştur.

Anahtar kelimeler: Yavaş gelişen piliçler, melezleme, et rengi, et pH'sı, karkas parçaları

\section{Introduction}

Intensive broiler meat production in the world is growing and broiler meat has an important share of total meat consumption. In addition to this, there are developments in new products and production systems in parallel with the changes in consumer demands. Consumers also accept to pay more money for poultry products from semi-intensive, extensive, free-range and organic systems. The demand for these products is increasing due to public opinion that they are produced in natural, healthier, and animal friendly systems (Yang and Jiang, 2005; Sarica and Yamak, 2010a).

Early slaughter age of chickens which are used in conventional production, metabolic disorders related to fast growth, and criticisms of seeing the conventional system as "production like factories" have led to new investigations. For this purpose, use of slow growing coloured feathered chickens fed with low-quality feed and slaughtered at a delayed age have become widespread (Rizzi et al., 2007; Dou et al., 2009;
Almeida and Zuber, 2010). Dark meat and skin colour and consumer preferences about the flavour are the main factors affecting choice of slow growing chicken strain (Zaho et al., 2007, Sarica et al., 2010). Slow growing chickens are more adapted for organic or freerange production systems; as they reach $2-2.5 \mathrm{~kg}$ live weight in 80-120 days. Fast growth may cause physiologic and metabolic disorders (Julian, 1993; Whitehead et al., 2003). Prevalence of disorders reduces if all body parts grow in harmony (Sarica et al., 2009; Sarica and Yamak, 2010a). With this aim, slow growing chickens have developed in the different regions of the world. "Assured chicken production" in United Kingdom, "Qualitat und Schereit" in Germany, "IKB Chicken" in Netherlands, "Label de Qualite Wallon and Belplume" in Belgium, "Polo Corral" in Spain, "Label Rouge" in France and "Three Yellow" in China are the best known slow growing chickens (Yang and Jiang, 2005; Magdelaine et al., 2008). The Label Rouge program has been a model for slow growing genotype breeding in all parts of the world. Label Rouge chickens 
are about two times more expensive than conventional chickens because production period is longer than conventional broilers and feed conversion ratios of conventional broilers are better than Label Rouge chickens. Despite this price they have reached 30\% share in the chicken market of France (Westgren, 1999; Fanatico and Born, 2002; Magdelaine et al., 2008). Europe is leading the production of slow growing commercial hybrids while Hubbard and Sasso are the best known commercial companies. These companies produce parents of different colour and quality, suitable for slow growing chickens.

Demand for natural or organically produced products has increased in recent years in Turkey. Nevertheless, it is important to use economically profitable chickens instead of low productive chickens in back yard poultry production system (Sekeroglu and Sarica, 2007). Furtherance, slow or medium growing chickens slaughtered at the ages of 56-84 days have been successfully used in organic and free-range meat production systems. Producing the parents of these kinds of chickens is possible with selection and breeding practices (Yang and Jiang, 2005). New lines and breeds could be improved by incorporation of commercial broiler breeders or heavy egg type parents and selection of local genotypes (Emmerson, 2003; Yang and Jiang, 2005; Sarica and Yamak, 2010a, b).

In this study, growth and carcass traits of slow growing parents were compared with commercial broilers and pure parents. Slow growing parents were produced by using two heavy egg type parents (BARII and RIRII) and fast growing ROSS parents. Thus, the broiler performance of the material produced by pure or twoway crossing was executed.

\section{Material and Method}

The study was aimed to improve slow growing parents by using two heavy egg type parents and a commercial broiler breeder. The trial was conducted at the University of Ondokuz Mayis Agricultural Faculty Research Farm, Samsun, Turkey from February 2010 to July 2010. The project was supported by the Scientific and Technological Research Council of Turkey (Project No:109O334). All procedures were approved by the local Ethical Committee of Ondokuz Mayis University for Experimental Animals. Rhode Island Red II (RIR II) and Barred Plymouth Rock II (BAR II) obtained from the Poultry Research Institute (Ankara, Turkey) were used as egg type parents. Commercial Ross broiler breeder genotype was purchased from Aviagen. Males of sire line and females of dam line were used as broiler parents. Four dam lines and three sire lines were produced by two-way crossing and selection in the live weights of egg type parents. RIR II and BAR II lines were selected according to live weight at the ages of 6,8 and 12 weeks and RIRII $\hat{x} \times$ ROSS 9 and BARII $\hat{\gamma} \times$ XROSS 9 crosses were produced as dam lines.

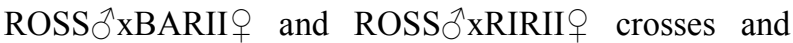
ROSS were used as sire lines.

A total of 144 male-female mixed chickens per genotype were reared on litter in a house divided into $1.5 \times 1.5 \mathrm{~m}$ pens. For each genotype, 4 replicates of 36 chickens in the same environment conditions were used in the experiment. Chicks were wing banded at hatching to determine sex and individual live weight differences.

A 23 hours light regime was applied in the first 6 weeks, with 14 hours lighting additional to natural lighting applied for the remainder of the experiment. Chickens were fed ad libitum as follows; 0-10 days: broiler starter diet (12.8 MJ ME/kg, 220g crude protein $/ \mathrm{kg}$ ), 11-25 days: broiler growing diet (13.2 MJ ME/kg, 200g crude protein $/ \mathrm{kg}$ ), 26 days to slaughter: broiler finisher diet (13.4 MJ ME/kg, 200g crude protein/kg). Feeds were purchased from a commercial mill. Chickens were vaccinated against New Castle and Gumboro diseases according to the advice of a local commercial broiler company. Feed consumptions and mortality were determined per pen. Feed conversion ratio (FCR) was calculated as feed intake divided by weight gain. Fast growing ROSS broilers were slaughtered at 46 days, while RIRII and BARII chickens were slaughtered at 84 days old age. Crossbred parents were slaughtered at three different ages (63, 70 and 84 days). Three male and three female chickens from each replicate were slaughtered on each slaughter day under similar conditions to minimize external factors. An $8 \mathrm{~h}$ fasting period was applied before slaughter, chickens were weighed individually at the plant where they were weighed and identified as male or female according to wing bands.

After slaughtering, carcasses were pre-chilled at $12{ }^{\circ} \mathrm{C}$ for $15 \mathrm{~min}$ and chilled at $4-5^{\circ} \mathrm{C}$ for $45 \mathrm{~min}$. After being chilled, carcasses were matured at $4^{\circ} \mathrm{C}$ for $12 \mathrm{~h}$, abdominal fat and chilled carcass weight was recorded, carcass weights, breast, back, wings, neck, legs (thighs and drumstick) were recorded (Sarica et al., 2009). Heart, liver and empty gizzard were weighed as edible inner organs. Carcass parts and edible inner organs were expressed as a percentage of chilled carcasses. Abdominal fat was expressed as a percentage of live weight. Shank lengths were measured as the distance 
between hock and foot pad of left legs. The skins of breast and thigh samples were stripped away, values of meat representing lightness $\left(\mathrm{L}^{*}\right)$, redness $\left(\mathrm{a}^{*}\right)$, and yellowness $\left(\mathrm{b}^{*}\right)$ were measured $12 \mathrm{~h}$ post-mortem using a Konica Minolta CR 400 Chroma Meter. Two replicate measures were made on both breast and thigh meats. The $\mathrm{pH}$ was measured with a $\mathrm{pH}$ meter (Model PC 510, Cyberscan, Singapore) equipped with an insertion electrode calibrated with $\mathrm{pH} 4.01$ and 7.01 buffers at ambient temperature. Three replicate measures were performed on breast and thigh meats.

Feed consumption and feed efficiency data were analysed by Analysis of variance and one way ANOVA model and other data were analysed by Analysis of variance and presented as consequence of each of the factors in the study: genotype, sex and genotype $\mathrm{x}$ sex interaction effects. Statistical analysis was conducted using the General Linear Model of SPSS software (SPSS Inc., 1999, Release 16.0). All percentage data were transformed by taking arcsine square roots prior to analysis. Mortality was analyzed by chi-square tests. When the F-test was significant, treatment means were compared using Duncan's multiple range tests. The level at which differences were considered significant was $\mathrm{P}<0.05$. Results are presented as means and a pooled SEM (unless otherwise stated).

\section{Results and Discussion}

Significant differences in live weights of the chickens among genotypes were found at various slaughtering ages $(\mathrm{P}<0.05)$. Fast growing ROSS genotype reached mean live weight of $2797.60 \mathrm{~g}$ at 46 days while crossbred genotypes reached these values at 70-84 days. RIRII and BARII genotypes never reached these values. It is expected that acceptable slow growing genotypes reach $2.5 \mathrm{~kg}$ in $80-85$ days. But some of the crossbred genotypes in this study reached marketing weight earlier than expected date (Table 1). Particularly, live weights of the ROSSxRIRII and ROSSxBARII crossbreds at 63 and 70 days showed that these two genotypes could be characterized as medium growing genotypes. These findings are similar to those of Fanatico et al. (2005a) who found $2.3-2.4 \mathrm{~kg}$ weight at 67 days. Berri et al. (2005) described chickens as medium growing chickens when they reached $2.65 \mathrm{~kg}$ at 8 weeks. Also, chickens that reached $2.10-2.88 \mathrm{~kg}$ at 12 weeks in the same study were termed as slow growing. In our study, the marketing weight of $2.7 \mathrm{~kg}$ obtained in the naturally mated RIRIIxROSS and BARIIxROSS genotypes could be acceptable for alternative production systems.

Dressing percentages of all genotypes were found to be between $70.17 \%$ and $73.85 \%(\mathrm{P}<0.05)$. These results are similar to the findings of Fanatico et al. (2005a), Abdullah et al. (2010) but greater than the findings of Dou et al. (2009). Dressing percentage increased with increasing age that was consistent with findings of Abdullah et el. (2010) but in contrast to Castellini et al. (2002a) (Table 1). Females had lower dressing percentage than males at all slaughter ages $(\mathrm{P}<0.05)$. Comparison of shank length to live weight (SL/LW) was given in Table 2. RIRII and BARII pure lines had the highest SL/LW values. These genotypes were egg type parents and layers have long shanks. It is thought that being egg type parents had effect on the highness of this value. Crossbred groups where egg type parents were used as sire lines had the second highest SL/LW values. The other crossbreds had higher values than ROSS genotype. While female chickens had shorter shanks; SL/LW value was found higher in females of some genotypes (Table 1).

Liver weight changed according to bodyweight of the bird and as an expected result, liver weight of the heavy groups was found to be heavier. RIRII and crossbred genotypes had higher gizzard weights. Edible inner organ ratios was found to be significantly greater in pure parents $(\mathrm{P}<0.05)$. The ratios of some body parts and carcass tissues changed with increased age (Murawska and Bochno, 2008). Contrary to this, there was a decrease with increased age in the crossbred groups of the study (Table 3 ). Gender had no significant effect on the edible inner organ ratios. It was previously reported by Berri et al. (2005) that in broilers, abdominal fat increased with increased production period. RIRII and BARII had the lowest abdominal fat percentage. ROSS chickens followed these genotypes. There were increases in the abdominal fat percentage of crossbred chickens with increased slaughter age $(\mathrm{P}<0.05$; Table 3). But, this increase reached about $5 \%$ of total body weight in the medium growing ROSSxRIRII and ROSSxBARII, while it was about $3 \%$ in slow growing groups at 84 days. In all genotypes, it was found that females had lower abdominal fat percentages than males $(\mathrm{P}<0.05)$.

While feed conversion ratios of fast growing chickens could be greater than 2.10 (Fanatico et al., 2005b), the ratio of 1.6 of ROSS chickens in the study could be accepted as a good result for conventional production systems. Crossbred chickens had different FCR at various ages. Feed conversion ratios increased with delayed slaughter age. These findings were in line with the results of Castellini et al. (2002a) who evaluated 
Table 1. Live weight and some carcass traits of genotypes at different slaughter ages.

\begin{tabular}{|c|c|c|c|c|c|c|c|c|}
\hline Genotype & Gender & $\begin{array}{c}\text { Age } \\
\text { (days) }\end{array}$ & $\begin{array}{l}\text { Live weight } \\
\text { (g) }\end{array}$ & $\begin{array}{l}\text { Carcass } \\
\text { weight }(\mathrm{g})\end{array}$ & $\begin{array}{l}\text { Dress. per. } \\
(\%)\end{array}$ & SL/LW & $\mathrm{EIO} / \mathrm{CW}$ & $\begin{array}{l}\text { Abdominal } \\
\text { fat }(\%)\end{array}$ \\
\hline \multirow{3}{*}{ RIRII } & $\mathrm{M}$ & 84 & 1870.8 & 1319.9 & 70.51 & 0.61 & 7.91 & 1.42 \\
\hline & $\mathrm{F}$ & & 1382.6 & 970.2 & 70.17 & 0.68 & 7.67 & 1.67 \\
\hline & $\mathrm{T}$ & & $1626.7 \mathrm{~h}$ & 1145.1 & $70.34 d$ & $0.65 \mathrm{a}$ & $7.79 a$ & $1.55 \mathrm{~g}$ \\
\hline \multirow{3}{*}{ BARII } & $\mathrm{M}$ & 84 & 1974.0 & 1397.0 & 70.77 & 0.61 & 5.95 & 2.13 \\
\hline & $\mathrm{F}$ & & 1416.0 & 997.2 & 70.42 & 0.71 & 5.99 & 2.03 \\
\hline & $\mathrm{T}$ & & $1695.0 \mathrm{~h}$ & $1161.1 \mathrm{~g}$ & $70.59 \mathrm{~d}$ & $0.66 \mathrm{a}$ & $5.97 \mathrm{~b}$ & $2.08 \mathrm{fg}$ \\
\hline \multirow{3}{*}{ ROSS } & $\mathrm{M}$ & & 3050.5 & 2180.9 & 71.48 & 0.36 & 5.63 & 1.91 \\
\hline & $\mathrm{F}$ & 46 & 2544.8 & 1814.6 & 71.31 & 0.34 & 5.85 & 2.51 \\
\hline & $\mathrm{T}$ & & $2797.6 b$ & 1997.8 & $71.40 \mathrm{~cd}$ & $0.35 \mathrm{~h}$ & $5.74 \mathrm{bc}$ & $2.21 \mathrm{f}$ \\
\hline \multirow{9}{*}{ ROSSxRIRII } & $\mathrm{M}$ & & 2334.2 & 1702.6 & 71.16 & 0.44 & 5.41 & 3.62 \\
\hline & $\mathrm{F}$ & 63 & 1859.4 & 1371.0 & 70.59 & 0.47 & 5.92 & 4.55 \\
\hline & $\mathrm{T}$ & & $2096.8 \mathrm{f}$ & $1536.8 \mathrm{ef}$ & $70.87 \mathrm{~cd}$ & $0.45 \mathrm{cde}$ & $5.67 \mathrm{bc}$ & $4.08 \mathrm{bc}$ \\
\hline & $\mathrm{M}$ & & 2617.6 & 1861.9 & 71.77 & 0.42 & 5.77 & 3.66 \\
\hline & $\mathrm{F}$ & 70 & 2021.2 & 1429.1 & 71.39 & 0.46 & 5.90 & 3.83 \\
\hline & $\mathrm{T}$ & & $2319.4 c$ & 1645.5 & $71.58 \mathrm{bcd}$ & $0.44 \mathrm{de}$ & $5.84 \mathrm{bc}$ & $3.74 \mathrm{~cd}$ \\
\hline & $\mathrm{M}$ & & 3376.7 & 2555.1 & 73.72 & 0.36 & 4.37 & 3.88 \\
\hline & $\mathrm{F}$ & 84 & 2496.8 & 1836.3 & 72.88 & 0.43 & 4.55 & 4.80 \\
\hline & $\mathrm{T}$ & & $2936.7 \mathrm{a}$ & $2195.7 b$ & $73.30 \mathrm{a}$ & $0.40 \mathrm{~g}$ & $4.46 \mathrm{e}$ & $4.34 \mathrm{ab}$ \\
\hline \multirow{9}{*}{ ROSSxBARII } & $\mathrm{M}$ & & 2233.2 & 1623.0 & 71.98 & 0.45 & 4.97 & 3.89 \\
\hline & $\mathrm{F}$ & 63 & 1789.2 & 1325.2 & 71.23 & 0.47 & 5.01 & 4.06 \\
\hline & $\mathrm{T}$ & & $2011.2 \mathrm{fg}$ & 1474.1f & $71.60 \mathrm{bcd}$ & $0.46 \mathrm{bcd}$ & $4.99 \mathrm{~d}$ & $3.97 b c$ \\
\hline & $\mathrm{M}$ & & 2548.9 & 1835.1 & 71.92 & 0.41 & 4.88 & 3.89 \\
\hline & $\mathrm{F}$ & 70 & 1998.9 & 1507.1 & 72.30 & 0.45 & 4.76 & 4.50 \\
\hline & $\mathrm{T}$ & & $2273.9 \mathrm{~cd}$ & $1671.1 \mathrm{~d}$ & $72.11 b c$ & $0.43 \mathrm{def}$ & $4.82 \mathrm{de}$ & $4.19 b$ \\
\hline & $\mathrm{M}$ & & 3400.4 & 2559.2 & 74.27 & 0.38 & 3.73 & 4.08 \\
\hline & $\mathrm{F}$ & 84 & 2476.3 & 1896.1 & 73.42 & 0.44 & 3.84 & 5.59 \\
\hline & $\mathrm{T}$ & & $2938.3 \mathrm{a}$ & $2227.6 a$ & $73.85 \mathrm{a}$ & $0.41 \mathrm{fg}$ & $3.79 \mathrm{f}$ & $4.84 \mathrm{a}$ \\
\hline \multirow{9}{*}{ RIRIIxROSS } & $\mathrm{M}$ & & 2288.8 & 1698.0 & 71.80 & 0.44 & 5.54 & 2.98 \\
\hline & $\mathrm{F}$ & 63 & 1675.1 & 1233.2 & 71.97 & 0.51 & 5.70 & 3.20 \\
\hline & $\mathrm{T}$ & & $1982.0 \mathrm{~g}$ & $1465.6 \mathrm{f}$ & $71.88 \mathrm{bc}$ & $0.48 \mathrm{~b}$ & $5.62 \mathrm{bc}$ & $3.09 \mathrm{e}$ \\
\hline & $\mathrm{M}$ & & 2555.7 & 1961.7 & 71.09 & 0.39 & 5.41 & 2.64 \\
\hline & $\mathrm{F}$ & 70 & 1794.9 & 1355.1 & 70.73 & 0.49 & 5.67 & 3.09 \\
\hline & $\mathrm{T}$ & & $2175.3 \mathrm{de}$ & $1658.4 d$ & $70.91 \mathrm{~cd}$ & $0.44 \mathrm{de}$ & $5.54 \mathrm{c}$ & $2.86 \mathrm{e}$ \\
\hline & $\mathrm{M}$ & & 3267.2 & 2225.5 & 72.89 & 0.39 & 4.52 & 2.70 \\
\hline & $\mathrm{F}$ & 84 & 2280.3 & 1676.1 & 72.62 & 0.47 & 4.49 & 3.80 \\
\hline & $\mathrm{T}$ & & $2773.7 \mathrm{bc}$ & $1950.8 \mathrm{c}$ & $72.75 \mathrm{ab}$ & $0.43 \mathrm{def}$ & $4.51 \mathrm{e}$ & $3.25 \mathrm{de}$ \\
\hline \multirow{9}{*}{ BARIIxROSS } & $\mathrm{M}$ & & 2239.7 & 1595.1 & 71.96 & 0.47 & 5.22 & 3.11 \\
\hline & $\mathrm{F}$ & 63 & 1729.0 & 1294.5 & 72.02 & 0.49 & 4.99 & 3.90 \\
\hline & $\mathrm{T}$ & & $1984.3 \mathrm{~g}$ & $1444.8 \mathrm{f}$ & $71.99 b c$ & $0.48 b$ & $5.11 \mathrm{~d}$ & $3.50 \mathrm{cde}$ \\
\hline & $\mathrm{M}$ & & 2532.8 & 1814.1 & 73.67 & 0.43 & 4.51 & 2.69 \\
\hline & $\mathrm{F}$ & 70 & 1876.8 & 1388.4 & 71.94 & 0.46 & 4.48 & 4.31 \\
\hline & $\mathrm{T}$ & & $2204.8 \mathrm{~cd}$ & $1601.2 \mathrm{de}$ & $72.80 \mathrm{ab}$ & $0.44 \mathrm{de}$ & $4.49 \mathrm{e}$ & $3.50 \mathrm{cde}$ \\
\hline & $\mathrm{M}$ & & 3236.3 & 2501.3 & 73.84 & 0.39 & 3.77 & 2.75 \\
\hline & $\mathrm{F}$ & 84 & 2291.9 & 1742.6 & 73.39 & 0.46 & 4.31 & 3.29 \\
\hline & $\mathrm{T}$ & & $2764.1 b$ & $2121.9 b$ & $73.61 \mathrm{a}$ & 0.43 def & $4.04 f$ & $3.02 \mathrm{e}$ \\
\hline SEM & & & 8.472 & 8.029 & 0.099 & 0.002 & 0.035 & 0.053 \\
\hline \multicolumn{9}{|l|}{ Effects } \\
\hline Genotype & & & $* *$ & $* *$ & $* *$ & $* *$ & $* *$ & $* *$ \\
\hline Sex & & & $* *$ & $* *$ & $* *$ & $* *$ & NS & $* *$ \\
\hline Genotype x Sex & & & $* *$ & $* *$ & NS & $* *$ & NS & NS \\
\hline
\end{tabular}

**:P<0.01; NS: Differences are insignificant, $\mathrm{P}>0.05 ; \mathrm{a}, \mathrm{b}, \mathrm{c}, \mathrm{d}, \mathrm{e}, \mathrm{f}, \mathrm{g}$ : Means with different letters in the same column are significantly different $(\mathrm{P}<0.05)$. SL/LW: Shank length/Live weight; EIO/CW: Edible inner organ/Carcass weight; Dress per: Dressing percentage; M: Male; F: Female; T: Female-Male mixed, SEM: Standard Error Mean

ROSSxRIRII and ROSSxBARII genotypes as medium growing chickens. Optimal slaughter age was found to be 63 or 70 days. These two genotypes had better feed conversion ratios than the findings of previous studies about medium growing chickens (Fanatico et al., 2005b; Castellini et al., 2002a). Feed conversion ratios at 84 days of both RIRIIxROSS and BARIIxROSS were found to be about 2.45, under the acceptable FCR 3.00- 
Table 2. Feed consumptions, feed conversion ratios and mortalities of different genotypes at different slaughter ages.

\begin{tabular}{lcllc}
\hline Genotypes & Age (days) & Feed Consumption $(\mathrm{g})$ & FCR & Mortality (\%) \\
\hline RIRII & 63 & $2493.3 \mathrm{j}$ & $1.95 \mathrm{i}$ & $1.70 \mathrm{a}$ \\
& 70 & $3221.7 \mathrm{i}$ & $2.35 \mathrm{ef}$ & $2.00 \mathrm{a}$ \\
& 84 & $4412.9 \mathrm{f}$ & $2.71 \mathrm{a}$ & $2.00 \mathrm{a}$ \\
\hline BARII & 63 & $2610.0 \mathrm{j}$ & $2.03 \mathrm{~h}$ & $2.70 \mathrm{a}$ \\
& 70 & $3326.7 \mathrm{i}$ & $2.40 \mathrm{cde}$ & $2.00 \mathrm{a}$ \\
\hline ROSS & 84 & $4605.4 \mathrm{e}$ & $2.72 \mathrm{a}$ & $2.00 \mathrm{a}$ \\
\hline ROSSxRIRII & 46 & $4511.7 \mathrm{ef}$ & $1.61 \mathrm{k}$ & $1.10 \mathrm{a}$ \\
& 63 & $3981.6 \mathrm{~g}$ & $1.90 \mathrm{j}$ & $1.10 \mathrm{a}$ \\
& 70 & $5406.2 \mathrm{c}$ & $2.33 \mathrm{f}$ & $1.80 \mathrm{a}$ \\
\hline ROSSxBARII & 84 & $7026.7 \mathrm{a}$ & $2.39 \mathrm{de}$ & $1.50 \mathrm{a}$ \\
& 63 & $3802.9 \mathrm{~h}$ & $1.89 \mathrm{j}$ & $1.60 \mathrm{a}$ \\
& 70 & $5167.8 \mathrm{~d}$ & $2.27 \mathrm{~g}$ & $0.00 \mathrm{~b}$ \\
\hline RIRIIxROSS & 84 & $6985.1 \mathrm{a}$ & $2.38 \mathrm{def}$ & $0.00 \mathrm{~b}$ \\
& 63 & $3894.7 \mathrm{gh}$ & $1.97 \mathrm{i}$ & $0.00 \mathrm{~b}$ \\
\hline BARIIxROSS & 70 & $5267.4 \mathrm{~d}$ & $2.42 \mathrm{bcd}$ & $0.00 \mathrm{~b}$ \\
& 84 & $6808.2 \mathrm{~b}$ & $2.45 \mathrm{bc}$ & $0.00 \mathrm{~b}$ \\
& 63 & 3904.8 gh & $1.97 \mathrm{i}$ & $0.00 \mathrm{~b}$ \\
\hline SEM & 70 & $5277.5 \mathrm{~d}$ & $2.39 \mathrm{de}$ & 0.001 \\
\hline Effect & 84 & $6818.3 \mathrm{~b}$ & $2.47 \mathrm{~b}$ & $* .039$ \\
\hline Genotype & 186.61 & $* *$ & \\
\hline Conversion Ratio, SEM: Standard Error Mean & & &
\end{tabular}

4.00 of slow growing chickens. This result was better than those of Castellini et al., (2002b) and Dou et al., (2009) who found values between 3.3 and 4.4. Pure lines RIRII and BARII had FCR higher than 2.45 but lower than 3.00. There was no significant mortality among all genotypes (Table 2).

Carcass part ratios of all genotypes at different slaughter ages are given in Table 3. Breast ratio, which is economically important, was found highest in fast growing ROSS genotype, ROSS x BARII and other crossbred chickens followed ROSS respectively. RIRII and BARII had the lowest breast ratio compared to other chickens. Breast ratios of the crossbred chickens were found between 28-31\% which can be defined as acceptable in commercial markets. Ratio of ROSS chickens is a result of breeding strategies applied for long times. Leg cut percentages of the genotypes were diametrically opposite to breast ratios. RIRII and BARII chickens had the heavier leg cut percentages compared to other genotypes. As an expected result, breast and leg cut percentages were found between the values of ROSS, RIRII and BARII. It can be said that crossbred chickens improved in this study and had the leg and breast ratios that were expected for slow growing chickens. The findings of this study are similar to those of Fanatico et al. (2005b), Berri et al. (2005), De Marchi et al. (2005) and Dou et al. (2009). Also, gender had a significant effect on breast-leg ratios $(\mathrm{P}<0.05)$. The other carcass parts (back, wings and neck) percentages were found lowest in ROSS genotype.

A significant effect of genotype was observed on $\mathrm{pH}$, $\mathrm{L}^{*}, \mathrm{a}^{*}$ and $\mathrm{b}^{*}$ values of breast and leg meat $(\mathrm{P}<0.01$, Table 4). Breast and leg meat of ROSS genotype had higher $\mathrm{L}^{*}$ values (paleness) than did those of RIRII and BARII genotypes, whereas crossbred genotypes had the lowest $\mathrm{L}^{*}$ values. However, breast and leg meat of ROSS genotype had the lowest a* value (redness) while crossbred genotypes had the highest. $b^{*}$ (yellowness) values were found highest in ROSS genotype's breast and leg meats. The leg muscle from BARII and crossbred genotypes had higher $\mathrm{pH}$ values than RIRII and ROSS genotypes. Breast muscle $\mathrm{pH}$ values were found higher in some crossbred groups. Particularly, highness of $\mathrm{a}^{*}$ value in the muscles of crossbred chickens is an expected quality trait in the meats of slow and medium growing chickens.

There is a negative correlation between the meat color and $\mathrm{pH}$ value of chicken meat. Meats with lower $\mathrm{pH}$ values have higher $\mathrm{L}^{*}$ values.

According to the results of this study, genotypes improved as parents could be used as slow or medium growing chickens. Particularly, carcass and growth performances of two-way crossbreds at different 
slaughter ages support this idea. Results of the second generation, chickens improved from the parents of this study, will execute healthier results about improving local slow growing chickens. According to whole results of the project, studies about these parents should be focus on reproductive traits such as; natural mating or artificial insemination, hatching properties and sexual maturation.

Table 3. The carcass weight (g) and parts cut-up characteristics (g/100g CW) of different genotypes

\begin{tabular}{|c|c|c|c|c|c|c|c|}
\hline \multirow{2}{*}{ Genotype } & \multirow{2}{*}{ Gender } & \multirow{2}{*}{ Age (days) } & \multicolumn{5}{|c|}{ Carcass Parts } \\
\hline & & & Leg & Breast & Back & Wings & Neck \\
\hline \multirow{3}{*}{ RIRII } & $\mathrm{M}$ & 84 & 34.88 & 23.58 & 20.76 & 12.87 & 7.31 \\
\hline & $\mathrm{F}$ & & 32.32 & 25.59 & 20.93 & 12.92 & 6.86 \\
\hline & $\mathrm{T}$ & & $33.60 \mathrm{a}$ & $24.59 \mathrm{~h}$ & 20.84 cde & $12.89 \mathrm{ab}$ & 7.08 cde \\
\hline \multirow{3}{*}{ BARII } & $\mathrm{M}$ & 84 & 34.18 & 25.69 & 21.24 & 11.59 & 6.79 \\
\hline & $\mathrm{F}$ & & 32.39 & 27.86 & 21.11 & 12.12 & 6.25 \\
\hline & $\mathrm{T}$ & & $33.28 \mathrm{ab}$ & $26.75 \mathrm{~g}$ & $21.17 \mathrm{~cd}$ & $11.86 \mathrm{fg}$ & $6.53 \mathrm{ef}$ \\
\hline \multirow{3}{*}{ ROSS } & M & & 30.28 & 34.96 & 17.67 & 10.46 & 6.25 \\
\hline & $\mathrm{F}$ & 46 & 29.04 & 35.83 & 18.21 & 10.72 & 6.29 \\
\hline & $\mathrm{T}$ & & $29.66 \mathrm{f}$ & $35.39 \mathrm{a}$ & $17.94 \mathrm{~g}$ & $10.59 \mathrm{i}$ & $6.27 \mathrm{f}$ \\
\hline \multirow{9}{*}{ ROSS x RIRII } & M & & 34.62 & 28.44 & 19.43 & 12.29 & 8.71 \\
\hline & $\mathrm{F}$ & 63 & 31.89 & 28.64 & 21.63 & 12.48 & 8.69 \\
\hline & $\mathrm{T}$ & & $33.26 \mathrm{abc}$ & $28.54 \mathrm{f}$ & 20.53 cde & $12.39 \mathrm{cde}$ & $8.70 \mathrm{a}$ \\
\hline & $\mathrm{M}$ & & 33.62 & 27.47 & 21.60 & 12.39 & 7.94 \\
\hline & $\mathrm{F}$ & 70 & 31.36 & 30.09 & 21.32 & 12.65 & 7.58 \\
\hline & $\mathrm{T}$ & & $32.49 \mathrm{abcd}$ & 28.78 ef & $21.46 \mathrm{bc}$ & $12.52 \mathrm{bcd}$ & $7.76 \mathrm{~b}$ \\
\hline & $\mathrm{M}$ & & 32.02 & 28.70 & 23.29 & 11.65 & 6.99 \\
\hline & $\mathrm{F}$ & 84 & 30.26 & 31.41 & 23.52 & 11.79 & 6.56 \\
\hline & $\mathrm{T}$ & & $31.14 \mathrm{e}$ & $30.06 \mathrm{~cd}$ & $23.41 \mathrm{a}$ & $11.72 \mathrm{gh}$ & $6.79 \mathrm{def}$ \\
\hline \multirow{9}{*}{ ROSS x BARII } & $\mathrm{M}$ & & 32.43 & 29.49 & 19.04 & 11.85 & 8.31 \\
\hline & $\mathrm{F}$ & 63 & 31.01 & 31.89 & 18.94 & 11.76 & 8.46 \\
\hline & $\mathrm{T}$ & & $31.72 \mathrm{de}$ & $30.69 \mathrm{bc}$ & $18.99 \mathrm{f}$ & $11.80 \mathrm{fgh}$ & $8.38 \mathrm{a}$ \\
\hline & $\mathrm{M}$ & & 33.45 & 27.86 & 19.66 & 12.36 & 8.94 \\
\hline & $\mathrm{F}$ & 70 & 30.68 & 31.62 & 20.39 & 12.04 & 8.31 \\
\hline & $\mathrm{T}$ & & $32.06 \mathrm{cde}$ & 29.74 cde & $20.03 \mathrm{def}$ & $12.20 \mathrm{cdef}$ & $8.62 \mathrm{a}$ \\
\hline & $\mathrm{M}$ & & 32.89 & 29.80 & 22.39 & 11.22 & 6.76 \\
\hline & $\mathrm{F}$ & 84 & 29.93 & 31.28 & 22.59 & 11.58 & 6.51 \\
\hline & $\mathrm{T}$ & & $31.41 \mathrm{de}$ & $30.54 \mathrm{bc}$ & $22.49 \mathrm{ab}$ & $11.39 \mathrm{~h}$ & $6.63 \mathrm{ef}$ \\
\hline \multirow{9}{*}{ RIRII x ROSS } & $\mathrm{M}$ & & 32.52 & 28.46 & 19.69 & 12.45 & 8.22 \\
\hline & $\mathrm{F}$ & 63 & 31.17 & 29.09 & 19.72 & 12.76 & 8.77 \\
\hline & $\mathrm{T}$ & & $31.85 \mathrm{de}$ & 28.77 ef & $19.71 \mathrm{ef}$ & $12.60 \mathrm{bc}$ & $8.49 \mathrm{a}$ \\
\hline & $\mathrm{M}$ & & 32.45 & 28.12 & 20.69 & 12.88 & 7.66 \\
\hline & $\mathrm{F}$ & 70 & 31.77 & 29.48 & 20.36 & 13.19 & 7.39 \\
\hline & $\mathrm{T}$ & & $32.11 \mathrm{bcde}$ & $28.80 \mathrm{ef}$ & 20.52 cde & $13.04 \mathrm{a}$ & $7.52 \mathrm{bc}$ \\
\hline & $\mathrm{M}$ & & 31.94 & 28.88 & 22.37 & 12.48 & 8.05 \\
\hline & $\mathrm{F}$ & 84 & 31.16 & 30.08 & 22.82 & 12.58 & 6.66 \\
\hline & $\mathrm{T}$ & & $31.55 \mathrm{de}$ & 29.48 def & $22.59 \mathrm{a}$ & $12.53 \mathrm{bcd}$ & $7.36 \mathrm{bcd}$ \\
\hline \multirow{9}{*}{ BARII x ROSS } & $\mathrm{M}$ & & 32.36 & 28.56 & 20.11 & 12.17 & 8.32 \\
\hline & $\mathrm{F}$ & 63 & 31.32 & 30.39 & 19.46 & 12.03 & 8.64 \\
\hline & $\mathrm{T}$ & & $31.84 \mathrm{de}$ & $29.47 \mathrm{def}$ & $19.78 \mathrm{ef}$ & 12.10 defg & $8.48 \mathrm{a}$ \\
\hline & $\mathrm{M}$ & & 32.36 & 29.72 & 20.42 & 12.19 & 7.84 \\
\hline & $\mathrm{F}$ & 70 & 31.82 & 28.08 & 19.64 & 11.83 & 7.03 \\
\hline & $\mathrm{T}$ & & 32.09 bcde & $28.90 \mathrm{ef}$ & $20.03 \mathrm{def}$ & $12.01 \mathrm{efg}$ & $7.44 \mathrm{bc}$ \\
\hline & M & & 32.67 & 29.86 & 21.98 & 11.96 & 6.29 \\
\hline & $\mathrm{F}$ & 84 & 30.89 & 32.33 & 22.99 & 11.64 & 6.49 \\
\hline & $\mathrm{T}$ & & $31.78 \mathrm{de}$ & $31.10 \mathrm{~b}$ & $22.49 \mathrm{ab}$ & $11.79 \mathrm{fgh}$ & $6.39 \mathrm{f}$ \\
\hline SEM & & & 0.100 & 0.085 & 0.096 & 0.037 & 0.050 \\
\hline \multicolumn{8}{|l|}{ Effects } \\
\hline Genotype & & & $* *$ & $* *$ & $* *$ & $* *$ & $* *$ \\
\hline Sex & & & $* *$ & $* *$ & NS & NS & $*$ \\
\hline Genotype x Sex & & & NS & $* *$ & NS & NS & NS \\
\hline
\end{tabular}

**:P<0.01;*:P<0.05 NS: Differences are insignificant, $\mathrm{P}>0.05$. a,b,c,d,e,f,g,h,i: Means with different letters in the same column are significantly different $(\mathrm{P}<0.05)$. M: Male; F: Female; T: Female-Male mixed; SEM: Standard Error Mean 
Table 4. $\mathrm{L}^{*}, \mathrm{a}^{*}, \mathrm{~b}^{*}$ and $\mathrm{pH}$ values of leg and breast meat of genotypes.

\begin{tabular}{|c|c|c|c|c|c|c|c|c|c|c|}
\hline \multirow{2}{*}{ Genotype } & \multirow{2}{*}{ Gender } & \multirow{2}{*}{$\begin{array}{c}\text { Age } \\
\text { (days) }\end{array}$} & \multicolumn{4}{|l|}{ Leg Meat } & \multicolumn{4}{|c|}{ Breast Meat } \\
\hline & & & $\mathrm{L}^{*}$ & $\mathrm{a}^{*}$ & $\mathrm{~b}^{*}$ & $\mathrm{pH}$ & $\mathrm{L}^{*}$ & $\mathrm{a}^{*}$ & $\mathrm{~b}^{*}$ & $\mathrm{pH}$ \\
\hline \multirow{3}{*}{ RIRII } & $M$ & 84 & 61.04 & 5.00 & 6.35 & 5.99 & 59.19 & 2.04 & 4.46 & 5.75 \\
\hline & $\mathrm{F}$ & & 60.93 & 5.16 & 9.85 & 5.67 & 60.49 & 2.29 & 6.92 & 5.48 \\
\hline & $\mathrm{T}$ & & $60.99 \mathrm{bc}$ & $5.08 \mathrm{c}$ & $8.09 \mathrm{~b}$ & $5.83 \mathrm{bc}$ & $59.84 \mathrm{~b}$ & $2.17 \mathrm{~d}$ & $5.69 \mathrm{~b}$ & $5.61 \mathrm{de}$ \\
\hline \multirow{3}{*}{ BARII } & $\mathrm{M}$ & 84 & 61.92 & 3.91 & 6.37 & 6.09 & 61.32 & 1.81 & 7.11 & 5.87 \\
\hline & $\mathrm{F}$ & & 61.07 & 3.97 & 7.72 & 5.79 & 58.90 & 1.26 & 7.92 & 5.58 \\
\hline & $\mathrm{T}$ & & $61.49 \mathrm{~b}$ & $3.94 \mathrm{~d}$ & $7.05 \mathrm{~b}$ & $5.94 \mathrm{ab}$ & $60.11 \mathrm{~b}$ & $1.54 \mathrm{e}$ & $7.51 \mathrm{a}$ & $5.72 \mathrm{~cd}$ \\
\hline \multirow{3}{*}{ ROSS } & $M$ & & 65.59 & 3.25 & 10.96 & 5.72 & 64.25 & 1.39 & 6.23 & 5.46 \\
\hline & $\mathrm{F}$ & 46 & 65.59 & 3.37 & 11.55 & 5.50 & 63.67 & 1.28 & 6.47 & 5.26 \\
\hline & $\mathrm{T}$ & & $65.59 \mathrm{a}$ & $3.31 \mathrm{~d}$ & $11.25 \mathrm{a}$ & $5.61 \mathrm{e}$ & $63.96 \mathrm{a}$ & $1.39 \mathrm{e}$ & $6.35 \mathrm{ab}$ & $5.36 \mathrm{f}$ \\
\hline \multirow{9}{*}{$\begin{array}{l}\text { ROSS x } \\
\text { RIRII }\end{array}$} & $\mathrm{M}$ & & 58.52 & 6.58 & 2.57 & 5.73 & 58.21 & 3.39 & 2.88 & 5.53 \\
\hline & $\mathrm{F}$ & 63 & 59.27 & 5.97 & 2.67 & 5.68 & 60.17 & 2.99 & 3.86 & 5.49 \\
\hline & $\mathrm{T}$ & & $58.89 \mathrm{de}$ & $6.28 \mathrm{ab}$ & $2.62 \mathrm{c}$ & 5.71cde & $59.19 b c$ & 3.19abc & $3.37 \mathrm{~cd}$ & $5.51 \mathrm{e}$ \\
\hline & $\mathrm{M}$ & & 59.07 & 6.16 & 3.46 & 5.95 & 54.89 & 2.99 & 3.68 & 5.77 \\
\hline & $\mathrm{F}$ & 70 & 58.57 & 5.42 & 2.97 & 5.91 & 52.98 & 2.98 & 2.17 & 5.71 \\
\hline & $\mathrm{T}$ & & $58.82 \mathrm{de}$ & $5.79 \mathrm{abc}$ & $3.21 \mathrm{c}$ & $5.93 \mathrm{ab}$ & $53.93 \mathrm{fg}$ & $2.98 \mathrm{bc}$ & $2.93 \mathrm{~cd}$ & $5.74 \mathrm{bc}$ \\
\hline & $\mathrm{M}$ & & 60.29 & 6.21 & 2.94 & 6.04 & 54.22 & 3.68 & 3.49 & 5.88 \\
\hline & $\mathrm{F}$ & 84 & 58.28 & 6.01 & 3.32 & 5.94 & 57.72 & 3.41 & 2.97 & 5.79 \\
\hline & $\mathrm{T}$ & & $59.29 \mathrm{cde}$ & $6.11 \mathrm{ab}$ & $3.13 c$ & $5.99 \mathrm{a}$ & $55.97 \mathrm{def}$ & $3.55 \mathrm{ab}$ & $3.23 \mathrm{~cd}$ & $5.84 \mathrm{abc}$ \\
\hline \multirow{9}{*}{$\begin{array}{l}\text { ROSS x } \\
\text { BARII }\end{array}$} & $\mathrm{M}$ & & 57.91 & 6.19 & 3.67 & 5.76 & 58.67 & 2.93 & 3.19 & 5.56 \\
\hline & $\mathrm{F}$ & 63 & 57.53 & 6.38 & 2.54 & 5.66 & 57.49 & 3.34 & 3.61 & 5.46 \\
\hline & $\mathrm{T}$ & & $57.72 \mathrm{def}$ & $6.28 \mathrm{ab}$ & $3.11 \mathrm{c}$ & 5.71cde & $58.08 \mathrm{bcd}$ & $3.13 \mathrm{abc}$ & $3.40 \mathrm{~cd}$ & $5.51 \mathrm{e}$ \\
\hline & $\mathrm{M}$ & & 57.82 & 6.39 & 3.17 & 5.97 & 52.55 & 3.46 & 3.07 & 5.80 \\
\hline & $\mathrm{F}$ & 70 & 58.04 & 5.05 & 3.12 & 5.93 & 53.17 & 3.13 & 2.79 & 5.74 \\
\hline & $\mathrm{T}$ & & 57.93def & $5.72 \mathrm{abc}$ & $3.14 \mathrm{c}$ & $5.95 \mathrm{ab}$ & $52.86 \mathrm{~g}$ & $3.29 \mathrm{ab}$ & $2.93 \mathrm{~cd}$ & $5.77 \mathrm{bc}$ \\
\hline & $M$ & & 59.12 & 6.68 & 3.34 & 6.08 & 52.72 & 3.65 & 3.48 & 5.89 \\
\hline & $\mathrm{F}$ & 84 & 57.84 & 6.03 & 2.67 & 5.96 & 54.12 & 3.39 & 2.03 & 5.69 \\
\hline & $\mathrm{T}$ & & $58.48 \mathrm{de}$ & $6.36 \mathrm{ab}$ & $3.01 \mathrm{c}$ & $6.02 \mathrm{a}$ & $53.42 \mathrm{fg}$ & $3.52 \mathrm{ab}$ & $2.76 \mathrm{~cd}$ & $5.79 \mathrm{abc}$ \\
\hline \multirow{9}{*}{$\begin{array}{l}\text { RIRII } x \\
\text { ROSS }\end{array}$} & $\mathrm{M}$ & & 57.65 & 6.63 & 3.55 & 5.77 & 59.23 & 3.18 & 3.99 & 5.55 \\
\hline & $\mathrm{F}$ & 63 & 57.57 & 6.16 & 4.54 & 5.67 & 56.64 & 3.19 & 4.51 & 5.47 \\
\hline & $\mathrm{T}$ & & 57.61def & $6.39 \mathrm{ab}$ & $4.05 \mathrm{c}$ & $5.72 \mathrm{cde}$ & $57.93 \mathrm{bcd}$ & 3.19abc & $4.25 c$ & $5.51 \mathrm{e}$ \\
\hline & $M$ & & 60.19 & 5.58 & 3.01 & 6.00 & 53.44 & 3.56 & 2.19 & 5.82 \\
\hline & $\mathrm{F}$ & 70 & 59.11 & 5.47 & 2.43 & 5.97 & 56.21 & 3.30 & 2.79 & 5.77 \\
\hline & $\mathrm{T}$ & & $59.65 \mathrm{bcd}$ & $5.53 \mathrm{bc}$ & $2.72 \mathrm{c}$ & $5.99 \mathrm{a}$ & $54.83 \mathrm{efg}$ & $3.43 \mathrm{ab}$ & $2.49 \mathrm{~d}$ & $5.79 \mathrm{abc}$ \\
\hline & $M$ & & 58.20 & 6.95 & 3.06 & 6.09 & 53.28 & 3.29 & 2.93 & 5.93 \\
\hline & $\mathrm{F}$ & 84 & 58.05 & 6.24 & 2.84 & 5.98 & 53.56 & 3.75 & 2.02 & 5.80 \\
\hline & $\mathrm{T}$ & & 58.13def & $6.59 \mathrm{a}$ & $2.95 \mathrm{c}$ & $6.03 \mathrm{a}$ & $53.42 \mathrm{fg}$ & $3.52 \mathrm{ab}$ & $2.48 \mathrm{~d}$ & $5.86 \mathrm{ab}$ \\
\hline \multirow{9}{*}{$\begin{array}{l}\text { BARII } x \\
\text { ROSS }\end{array}$} & $\mathrm{M}$ & & 55.67 & 6.29 & 3.18 & 5.82 & 56.75 & 3.30 & 3.23 & 5.61 \\
\hline & $\mathrm{F}$ & 63 & 56.77 & 6.46 & 4.17 & 5.72 & 57.56 & 3.67 & 4.61 & 5.55 \\
\hline & $\mathrm{T}$ & & $56.22 \mathrm{fg}$ & $6.37 \mathrm{ab}$ & $3.67 \mathrm{c}$ & $5.77 \mathrm{~cd}$ & $57.16 \mathrm{cde}$ & $3.49 \mathrm{ab}$ & $3.92 \mathrm{~cd}$ & $5.58 \mathrm{e}$ \\
\hline & M & & 58.28 & 5.71 & 2.83 & 6.04 & 53.22 & 3.07 & 2.17 & 6.04 \\
\hline & $\mathrm{F}$ & 70 & 52.10 & 5.84 & 2.18 & 5.99 & 56.75 & 2.21 & 3.78 & 5.78 \\
\hline & $\mathrm{T}$ & & $55.19 \mathrm{~g}$ & $5.78 \mathrm{abc}$ & $2.51 \mathrm{c}$ & $6.02 \mathrm{a}$ & 54.99efg & $2.64 \mathrm{~cd}$ & $2.97 \mathrm{~cd}$ & $5.91 \mathrm{a}$ \\
\hline & $\mathrm{M}$ & & 57.35 & 6.32 & 2.45 & 6.09 & 54.50 & 3.96 & 3.62 & 5.89 \\
\hline & $\mathrm{F}$ & 84 & 57.45 & 6.30 & 2.30 & 6.01 & 51.85 & 3.40 & 2.65 & 5.81 \\
\hline & $\mathrm{T}$ & & $57.40 \mathrm{ef}$ & $6.31 \mathrm{ab}$ & $2.37 \mathrm{c}$ & $6.05 \mathrm{a}$ & $53.18 \mathrm{~g}$ & $3.68 \mathrm{a}$ & $3.13 \mathrm{~cd}$ & $5.85 \mathrm{abc}$ \\
\hline SEM & & & 0.173 & 0.073 & 0.197 & 0.010 & 0.221 & 0.049 & 0.125 & 0.011 \\
\hline \multicolumn{11}{|l|}{ Effects } \\
\hline Genotype & & & $* *$ & $* *$ & $* *$ & $* *$ & $* *$ & $* *$ & $* *$ & $* *$ \\
\hline Sex & & & $*$ & NS & NS & $* *$ & NS & NS & NS & $* *$ \\
\hline Genotype $\mathrm{x}$ & & & NS & NS & NS & $* *$ & NS & NS & NS & NS \\
\hline
\end{tabular}

$* *: \mathrm{P}<0.01 ; *: \mathrm{P}<0.05$ NS: Differences are insignificant, $\mathrm{P}>0.05$. a,b,c,d,e,f,g: Means with different letters in the same column are significantly different $(\mathrm{P}<0.05)$. M: Male; F: Female; T: Female-Male mixed, SEM: Standard Error Mean

\section{References}

Abdullah, A.Y., Al-Beitawi, N.A., Rjoup, M.M.S., Qudsieh R.J., Ishmais, M.A.A. 2010. Growth performance, carcass and meat quality characteristics of different commercial crosses of broiler strains of chickens. Journal of Poultry Science 47: 13-21.

Almeida, A.M., Zuber, U. 2010. The effect of the Naked Neck genotype (NAna), feding and outdoor rearing on growth and carcass characteristics of free range 
broilers in a hot climate. Tropical Animal Health \& Production 42: 99-107.

Berri, C., Le Bihan-Duval, E., Baeza, E., Chartrin, P., Picgirard, L., Jehl, N., Quentin, M., Picard, M., Duclos, M.J. 2005. Further processing characteristics of breast and leg meat from fast-, medium- and slow-growing commercial chickens. Animal Research 54: 123-134.

Castellini, C., Mugnai, C., Dal Bosco, A. 2002a. Effect of organic production system on broiler carcass and meat quality. Meat Science 60: 219-225.

Castellini, C., Dal Bosco, A., Mugnai, C., Bernardini, M. 2002b. Performance and behaviour of chickens with different growing rate reared according to the organic system. Italian Journal of Animal Science 1:291-300.

De Marchi, M., Cassandra, M., Lunardi, E., Baldan, G., Siegel, P.B. 2005. Carcass characteristics and qualitative meat traits of the Padovana breed of chicken. International Journal of Poultry Science 4: 233-238.

Dou, T.C., Shi, S.R., Sun, H.J., Wang, K.H. 2009. Growth rate, carcass traits and meat quality of slowgrowing chicken according to three raising systems. Animal Science Paper and Reports 27: 361-369.

Emmerson, D. 2003. Breeding objectives an selection strategies for broiler production. Poultry Genetics, Breeding and Biotechnology, Edited by W.M. Muir \& S.E., pp 113-127, Aggrey.

Fanatico, A.C., Born, H.M. 2002. Label Rouge: PastureRaised Poultry in France. ATTRA Publication National Centre for Appropriate Technology, Fayetteville.

Fanatico, A.C., Cavitt, L.C., Pillai, P.B., Emmert, J.L., Owens, C.M. 2005a. Evaluation of slower- growing broiler genotypes grown with and without outdoor Access:Meat Quality. Poultry Science 84: 17851790 .

Fanatico, A.C., Pillai, P.B., Cavitt, L.C., Owens, C.M., Emmert, J.L. 2005b. Evaluation of slower-growing broiler genotypes grown with and without outdoor Access:Growth performance and carcass yield. Poultry Science 84: 1321-1327.

Julian, R.J. 1993. Ascites in poultry. Avian Pathol. 22: 419-454.

Magdelaine, P., Spiess, M.P., Valceschini, E. 2008. Poultry meat consumption trends in Europe. World's Poultry Science Journal 64: 53-63.
Murawska, D., Bochno, R. 2008. Age-related changes in the percentage content of tissue components in geese. Journal of Central European Agriculture 9: 211-216.

Rizzi, C., Marangon, A., Chiericato, G.M. 2007. Effect of genotype on slaughtering performance and meat physical and sensory characteristics of organic laying hens. Poultry Science 86: 128-135.

Sarica, M., Ocak, N., Karacay, N., Yamak, U., Kop, C., Altop, A. 2009. Growth, slaugter and gastro intestinal tract traits of three turkey genotypes under barn and free range housing systems. British Poultry Science 50: 487-494.

Sarica, M., Yamak, U.S. 2010a. Developing slow growing meat chickens and their properties. Anadolu Tarım Bilimleri Dergisi 25: 61-67 (in Turkish).

Sarica, M., Yamak, U.S. 2010b. Organik ve ekstansif üretimde kullanılan yavaş gelişen etlik piliçlerin özellikleri ve geliştirilmesi. Türkiye IV. Organik Tarım Sempozyumu, Erzurum, pp 269-274 (in Turkish).

Sarica, M., Yamak, U.S., Boz, M.A. 2010. Growth and carcass characteristics of genotypes used as parent line for developing slow growing meat type parents. Proceeding XIII th European Poultry Conference, Tours pp 595.

Sekeroglu, A., Sarica, M. 2007. Alternatif Üretim Metodu Olarak Köy Tavukçuluğu. V. Ulusal Zootekni Ulusal Bilim Kongresi, Van (in Turkish).

SPSS, 1999. SPSS Release 16.0. SPSS Inc., Chicago, IL.

Westgren, R.E. 1999. Delivering food safety, food quality and sustainable production practices: The Label Rouge poultry system in France. American Journal of Agricultural Economics 81: 1107- 1111.

Whitehead, C.C., Fleming, R.H., Julian, R.J. 2003. Skeletal problems associated with selection for increased production (Ed. W.M., Muir, S.E., Aggrey) Poultry Genetics, Breeding and Biotechnology, 29-52. CABI Publishing, Cambridge, USA.

Yang, N., Jiang, R.S. 2005. Recent advances in breeding for quality chickens. World's Poultry Science Journal. 61: 373-381.

Zaho, G.P., Chen, J.L., Zheng, M.Q., Wen, J., Zhang, Y. 2007. Correlated responses to selection for increased intramuscular fat in a Chinese quality chicken line. Poultry Science 86: 2309- 2314. 